\title{
An improved technique for creating hemifacial chimeras
}

\author{
ROTEM KOWNER \\ University of Tsukuba, Tsukuba, Japan
}

\begin{abstract}
In numerous studies, facial composites of the right and left hemifaces have been used to explore lateral differences in facial expressions. In the majority of these studies, a single technique has been used to create the stimuli: "Wolff's split half technique," which was devised at the beginning of the century. Chimeras thus made are weakened by several confounding factors (e.g., hair form, background, shadows), which are unrelated to the original stimuli and consequently may interfere with the examination of subtle facial expressions. This paper offers a modified method using computer graphics that seems to solve such drawbacks.
\end{abstract}

Since the beginning of this century, there have been many investigations of the difference between the facial expressions of the right and left hemifaces as perceived by viewers. Whereas early studies were concentrated on the examination of differential representation of personality in each hemiface, in later studies this research has been pursued in an attempt to illuminate brain mechanisms in general, and hemispheric specialization in particular (for a review, see Campbell, 1986; Hager, 1982).

To create facial composites for stimulus presentation, researchers in the majority of the studies on facial asymmetry have employed a technique originally used by Hallevorden (1902, 1929) and Wolff (1933): one takes still photos of faces, makes one normal print and another print by turning the negative over, and cuts the final image and the mirror image along the midline. Then, one rejoins the corresponding halves to produce two composite photos, the one made solely of the left side of the face, and the other of the right side. Although some methodological advancements have been made over the years (e.g., hiding the hair), the basic technique has not changed since the first study. The success of this method (often called "Wolff's split half technique") can be attributed to the fact that it has been the only one to allow a thorough examination of the whole face in static and carefully arranged poses. In addition, it distinctly separates the two hemifaces during evaluation and eliminates the effect of the left bias that occurs in the perception of ordinary faces (Gilbert \& Bakan, 1973). Despite its merits, Wolff's split half technique suffers from several drawbacks that can lead to errors in the assessment of hemifacial differences and thus call into question many of the findings obtained by researchers who use it.

The author would like to thank Toshiki Ogawa and several anonymous reviewers for their comments on an earlier draft. Correspondence concerning this article should be addressed to R. Kowner, Department of Psychology, The Hebrew University, Mt. Scopus, Jerusalem 91905, Israel.
The first problem concerns the stimuli's hair. Duplicating the hair contour tends to create ridiculous and divergent hair forms in each composite, which lead to unnatural and magnified differences between the two composites. Likewise, unilateral shadows on the face, cast by inappropriate lighting, can cause the shadowy side to look unnaturally "darker" and thinner after duplication. Inadequate lighting can lead to a repeated bias in a series of pictures, while natural and unnatural blemishes are also asymmetrically duplicated. In addition, faces not positioned to be looking straight ahead (in the camera direction), as a result of habitual or personal inclination of the model, lead to an ostensibly different facial width and shape following duplication. Inadequate frontal lighting causes such deflections to cast larger shadows on the side that is farther from the camera. Finally, clothes, accessories, and other background cues tend to add an uneven subliminal effect, and the visible midline of the constructed pictures can indicate an experimenter's underlying intentions. Hence these drawbacks form various artificial and exaggerated differences between the two hemifaces and thus call into question the validity of this technique.

\section{An Improved Technique for Creating and Presenting Chimeric Faces}

To avoid the preceding faults, the following improved technique for creating hemifacial composites is proposed. The technique resembles the old manual method and yet avoids most of its drawbacks. In addition, it is easy to use and can be performed with various graphic software applications.

The minimum requirement is a Macintosh computer (System 6 or 7 with at least 2-4 Mb RAM, depending on the software used and the size of the pictures manipulated) and a graphics program. We used and recommend SuperPaint 3.0 graphics software (Silicon Beach Software, Inc.), but other software such as MacPaint or Canvas may do as well. Similarly, this procedure can be performed with any PC that has adequate memory, using 
one of the more recent versions of almost any graphics software with a paint mode.

The following steps serve as a general outline for the use of the modified technique with any software. The computer commands that are listed (in italics) can be found in most graphics programs, regardless of the computer, and often appear under the same name.

1. Scan the desired photo, with any scanning program (monochrome, grayscale, or color can be used). The larger the picture and the greater the scanning magnification, the better will be the quality of the picture when printed. Nevertheless, the size of the screen should be the guide for the upper limit of the picture size, because images much bigger than the screen size may be difficult to manipulate.

2. Open the file from the graphics program and convert it into a PICT format in the Paint mode (Figure 1, picture 1).

3. Delete all background details from the picture, using the Eraser and, if necessary, one pixel white Brush when dealing with fine details. Similarly, delete facial accessories (e.g., earrings), hair (e.g., pieces difficult to manipulate), and certain minor asymmetric blemishes. Finally, delete the part below the jaw line, because differing neck thickness may have a confounding effect on the observers' judgment (picture 2).
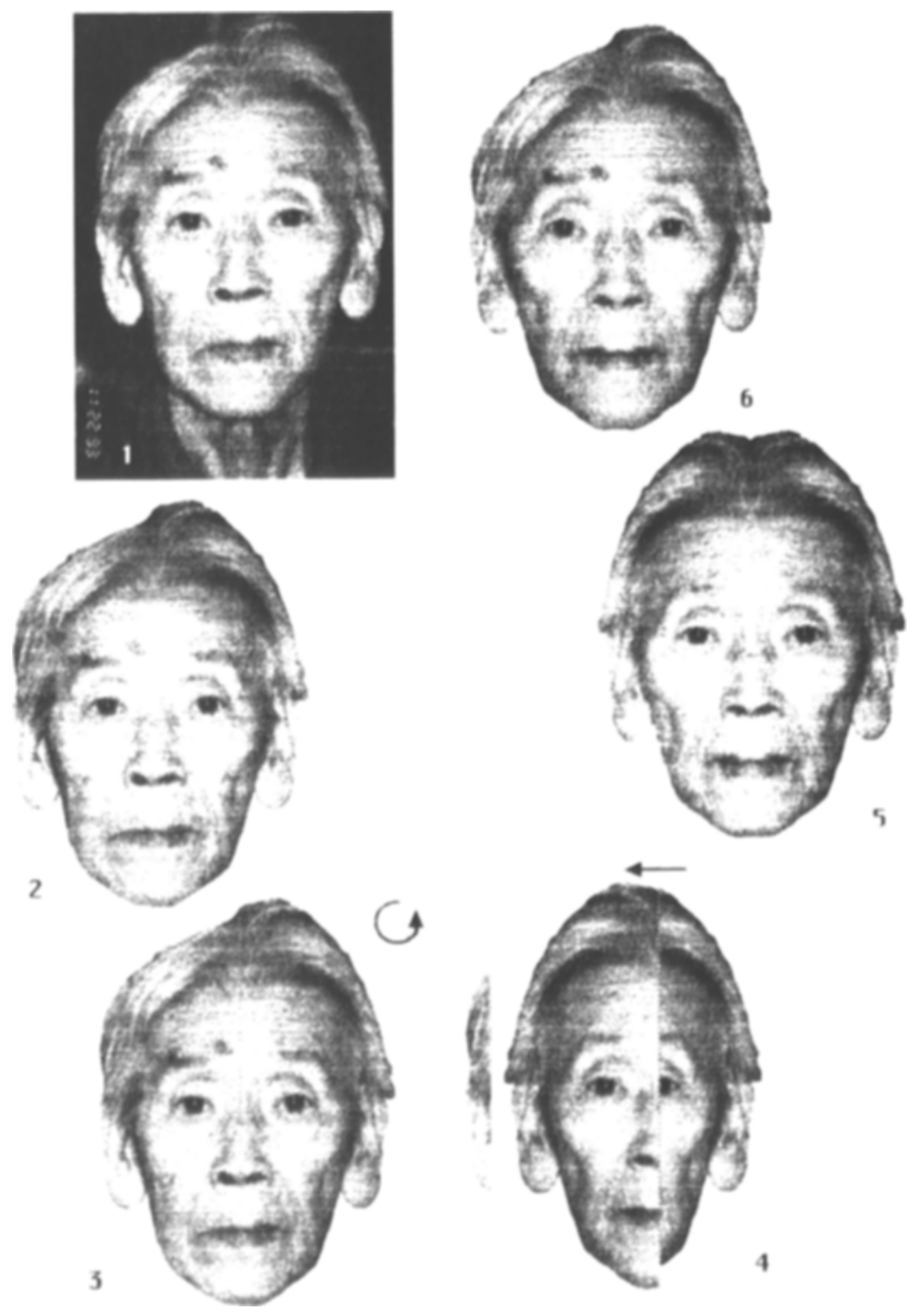

Figure 1. How to use the improved technique to create a left hemifacial chimera-a step by step guide: (1) The original photograph. (2) Aligning the portrait and deleting the background. (3) Flipping the left hemiface. (4) Moving the flipped left hemiface to its new position. (5) Placing it at the correct place. (6) Reconstructing the hairline and adding/deleting various facial marks. 
4. In case the head inclination is not perfectly horizontal, it may be necessary to align it with the Free rotate command (picture 2).

5 . When the only picture on the screen is the head surrounded by a white background, make a copy of the original portrait (Save as ...), and rename it (e.g., "left hemifacial composite"). Repeat the procedure for the "right hemifacial composite."

6. For the "left hemifacial composite," select the left half of the portrait (the part on the right side of the picture) and locate the center of the face, using the vertical midline connecting the midpoints between the eyes, nose, upper lip, and chin (picture 3).

7. Copy this selection, Paste it, and then reverse it by using the command Flip horizontal.

8. Move the flipped hemiface to its new position on the left side of the portrait. To keep the transferred half in its vertical position, press Shift while moving the hemiface. When the cut hemiface reaches its correct place, a special symmetric pattern will be formed between the two halves (pictures 4-5).

9. Select and Copy the hair pattern of the covered hemiface, and Paste it on the duplicated hemiface. Correct minor hair and shadow improprieties with the brush or pasted texture from the original portrait (use the Zoom in for fine corrections). Erase duplicated facial marks and blemishes, and similarly add marks from the original picture that have been lost during the duplication process (picture 6).

10. Repeat the same procedure (Steps 5-9) for the "right hemifacial composite."

The final pictures can be presented on a computer screen, printed, or used in slides. Users should keep in mind, however, that despite the capabilities of this method, it cannot "save" pictures that are not taken correctly. In other words, special care should be taken to avoid facial deflection and unbalanced illumination during the photographing session.

\section{Validity and Usage}

Is the preceding method really superior to "Wolff's split half technique"? Its first obvious advantage is its ease of use. Portraits can be easily manipulated with the software suggested above, so changes of position or size can be made in a few seconds. Also, if a computer is used, it is possible to benefit from the availability of highresolution printers to produce cheap, high-quality pictures quickly. Alternatively, a researcher may choose to display the portraits on a computer monitor and to record subjects' ratings directly into the computer by using specialized programs such as SuperLab or MacProbe (for reviews, see Haxby, Parasuraman, Lalonde, \& Abboud, 1993; Hunt, 1994). Finally and most important, Kowner (1995) has demonstrated that judgments of portraits made by this method may differ from judgments of portraits made with Wolff's split half technique. In this study (Experiment 2), three groups of subjects were shown 10 photos of either right- or left-hemifacial chimera com- posites of left-handed people and were asked to rate them on nine scales of 7-point bipolar adjective pairs concerning emotional expression and personality. The first two groups of subjects examined composites created by the new method, and a third group examined composites of the same stimulus person made with the "old" method. Subjects in the first two groups judged the portraits to have significant differences between the right and left hemifaces, which corroborated earlier findings of similarity between the two hemifaces in resting faces. In the third group, however, subjects judged the composites to have few differences, which indicated the potential for random attributional error with the old method. Moreover, subjects thought, when presented with both composites in a follow-up interview, that some of the pairs belonged to different people. This misattribution did not occur with composites created with the improved technique (see, e.g., Figure 2).

In six experiments, Kowner (1995) investigated whether there are consistent differences between the emotions attributed to the right and left hemifaces. Using the technique presented above, Kowner suggested that no consistent differences between the emotions and personality are attributed to the right and the left hemiface. Nevertheless, when the two hemifaces were simultaneously compared for intensity, the left hemiface showed a greater intensity in posed smiles. The study thus suggested the possibility of a slight inference of brain laterality in posed expressions, but not in resting faces.

I am convinced that the new technique can be used for any comparisons of the two lateral hemispheres of the face. The researcher who is interested in presenting the exact mirror image of a face should skip Step 9. Other variants of this technique that retain its basic aspects are also possible. Heller and Levy (1981), for example, tachistoscopically presented photographs of facial composites; half of the faces were smiling and the other half were not. Both right- and left-handed subjects perceived a face as happier when the left side of the poser's face was smiling. The stimulus persons in this study could have easily been prepared with the new technique, with two portraits of smiling and nonsmiling people. One need only exchange either the right or the left hemiface between the two portraits without flipping them. In the second stage, one should make a copy of the two portraits (Save as ...) and flip them horizontally. With this method a researcher can produce the four chimeras presented by Heller and Levy (p. 265) in a few minutes.

Another field in which the new method can be used is research on the role of asymmetry in facial attribution. Kowner (1994) examined the effect of hemifacial size on attributions of personality and emotion in normal people with pronounced facial asymmetry. Comparisons of 10 attributions made to the larger versus smaller hemifacial composites of the same face in 14 portraits of resting faces showed that the smaller hemifaces were rated as exhibiting more intense emotions and as generally having more positive features than were the larger hemi- 

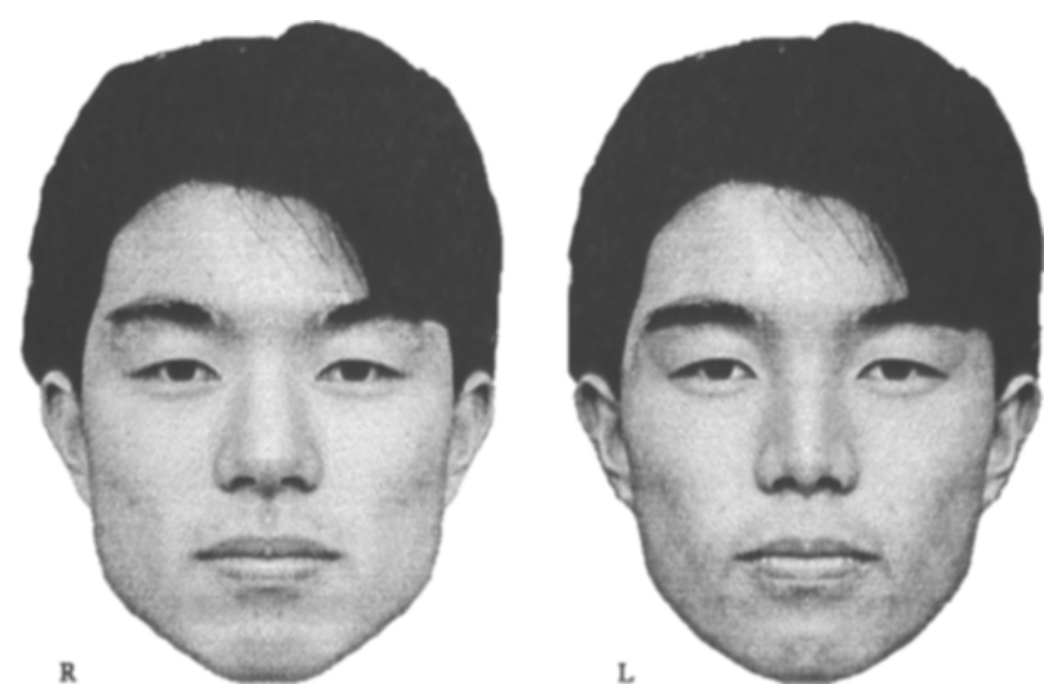

Figure 2. Composite chimeras of the right $(R)$ and left $(L)$ hemifaces (note that the left hemiface is slightly darker than the right one, owing to imperfect illumination that could not be corrected).

faces. The main implication of these findings is that faces with prominent asymmetry do not uniformly affect observers, but rather depend on the features of the hemiface perceived by the dominant visual field of the observers (generally the left one). This method can also be used for research on the role of facial symmetry in the judgment of physical attractiveness. Kowner (1996) has investigated people's preferences for either somewhat asymmetrical portraits or their symmetrical chimeric composites when presented simultaneously. Overall, this study suggests that the low degree of facial asymmetry found in normal people does not affect attractiveness ratings (except in the case of old people), probably because observers are not attuned to perceive it.

Finally, this new technique is not limited only to the research arena. Practitioners of plastic surgery, for example, can manipulate photos of patients with extreme facial asymmetry, and show them the possible outcomes of reconstructive operations (see Reid \& Farkas, 1994).

\section{REFERENCES}

Campbell, R. (1986). Asymmetries of facial action: Some facts and fancies of normal face movement. In R. Bruyer (Ed.), The neuropsychology of facial perception and facial expression (pp. 247. 267). Hillsdale, NJ: Erlbaum.

GILBERT, C., \& BAKAN, P. (1973). Visual asymmetry in perception of faces. Neuropsychologia, 11, 355-362.

Hager, J. C. (1982). Asymmetries in facial expression. In P. Ekman
(Ed.), Emotion in the human face (pp. 318-352). Cambridge: Cambridge University Press.

Hallevorden, J. (1902). Eine neue Methode experimenteller Physiognomik [A new method of experimental physiognomy]. PsychiatrischNeurologische Wochenschrift, 4, 309-311.

HALLEVORDEN, J. (1929). Rechts- und Links-handigkeit und Gesichtausdruck [Right- and left-handedness and facial expression]. Zentralblatt für die gesamte Neurologie und Psychiatrie, 53, 560.

Haxby, J. V., Parasuraman, R., Lalonde, F., \& Abboud, H. (1993). SuperLab: General-purpose Macintosh software for human experimental psychology and psychological testing. Behavior Research Methods, Instruments, \& Computers, 25, 400-405.

Heller, W., \& LeVY, J. (1981). Perception and expression of emotion in right-handers and left-handers. Neuropsychologia, 19, 263-267.

HuNT, S. M. J. (1994). MacProbe: A Macintosh-based experimenter's workstation for the cognitive sciences. Behavior Research Methods, Instruments, \& Computers, 26, 345-351.

KOWNER, R. (1994). The perception and attribution of hemifacial size in normal adults. Manuscript submitted for publication.

KowNER, R. (1995). Laterality in facial expressions and its effect on attributions of emotion and personality: A reconsideration. Neuropsychologia, 33, 539-559.

KOWNER, R. (1996). Facial asymmetry and attractiveness judgment in developmental perspective. Journal of Experimental Psychology: Human Perception \& Performance, 22, 662-675.

REID, P. S., \& FARKAS, L. G. (1994). Medical photography in clinical practice. In L. G. Farkas (Ed.), Anthropometry of the head and face (pp. 191-200). New York: Raven Press.

WOLFF, W. (1933). The experimental study of forms of expression. Character \& Personality, 2, 168-176.

(Manuscript received November 7, 1994; revision accepted for publication April 7, 1995.) 